\title{
Coordinated Excitation and Speed Robust Control Based on the State Extend and Filtering Estimation
}

\author{
Zhifeng $\mathrm{Gu}^{1, *}$, Changqing Zhu ${ }^{1}$, Chenguang Zhang ${ }^{1}$, Lijuan Liang ${ }^{2}$ and Qiaojing $\mathrm{An}^{1}$ \\ ${ }^{1}$ Vehicle and Electric department of Mechanical Engineering College, Shi Jiazhuang 050003, China \\ ${ }^{2}$ Physics department of Xing Tai University, Xing Tai 054001, China \\ ${ }^{*}$ Corresponding author
}

\begin{abstract}
For the nonlinear robust control difficulty in the MIMO system, which has many uncertain parameters, the state extending and filtering robust control (SEFERC) is proposed. The uncertain nonlinear part of the MIMO is extend to the new state parameter and can be estimated. With the estimated value, combined with the direct feedback linearization (DFL), the nonlinear system can convert to the linear system. According $\boldsymbol{H}_{\infty}$ control method, the robust control of the linear system is realized. Taking the certain excitation and speed coordinated control system for example, simulation results show that the SEFERC can accelerate the convergence speed of the state variables and can improve the excitation and speed coordinated control speed as illustrated by the portions given in this document.
\end{abstract}

Keywords- MIMO system; multi uncertain parameters; state extend; filtering estimation; nonlinear robust control; state parameter estimation

\section{INTRODUCTION}

Multi-machine power system has many characters, such as the complex structure, the nonlinearity and the parametric uncertainty. When there are many uncertain parameters, the control law design of the power system will be difficult. The multi-machine power system is one kind of the MIMO systems It is very significant to study the design of the control law for the multi-uncertain-parameters nonlinear MIMO system ${ }^{[1]-[2]}$.

In the nonlinear control, the exact feedback linearization (EFL) and the nonlinear control based on the objective holographic feedbacks (NCOHF) are proposed and can realize the linearization and the global asymptotic stability. But the realization of the EFL and the NCOHF need know the accurate model of the MIMO system ${ }^{[3]-[4]}$. The coordinated passivity control method, which based on the passivity theory and the back-stepping design technique, has the special requirement for the structure of the control system ${ }^{[5]}$. The method based on the Hamilton energy theory resolve the designing difficulty of the Lyapunov function, but at the same time the design of the Hamilton energy function is complicated ${ }^{[6]-[7]}$.

The uncertainty includes the inside uncertainty and the outside uncertainty. The inside uncertainties result from the variation in the structure and the parameter of the system. The outside uncertainties result from the extant disturbance. In order to overcome the influence from the uncertainties, the $\mathrm{H} \infty$ control, the sliding model control, the ESO control is proposed. These methods based on the stability theory and have some shortcomings, such as difficulties in the sliding model face selection, the chattering of the sliding model control, the complexity of the robust adaptive deduction and so on ${ }^{[8]-[10]}$. In recent yeas, many filtering estimation methods, such as EKF, $\mathrm{UKF}, \mathrm{CDKF}, \mathrm{STF}$ and so on ${ }^{[11]-[13]}$, develop rapidly and are used to estimate the values of the state parameters in the state equations. These methods are based on the filtering theory and the statistic theory and can overcome the uncertainty of the nonlinear system.

In this paper, combining the state extending technology, direct feedback linearization (DFL) and nonlinear state estimation, the state extend and filtering estimation robust control (SEFERC) method is proposed. In the SEFERC, the nonlinear uncertain parts of the state equation are extended to the new variables and estimated by nonlinear filtering method. The SEFERC is one kind of new method for the robust control. In the end of this paper, taking the certain excitation and speed coordinated control system for example, simulation results show that in comparison with PID-PSS control the SEFERC can accelerate the convergent speed of the state variables and can improve the excitation and speed coordinated control speed.

\section{EXCITATION AND SPEED COORDINATED SEFERC}

Mathematical model of the excitation and speed control system, which is of uncertainty, is shown as following equ.1.

$$
\begin{aligned}
& \dot{x}_{1}=x_{3} \\
& \dot{x}_{2}=-\beta_{1} x_{2}+\beta_{2} u_{1} \\
& \dot{x}_{3}=\beta_{3} x_{2}-\beta_{4} x_{3}-\beta_{5} \sin \left(\delta_{0}+x_{1}\right) x_{4}-\beta_{5} x_{40}\left[\sin \left(\delta_{0}+x_{1}\right)-\sin \left(\delta_{0}\right)\right] \\
& \dot{x}_{4}=-\beta_{6} x_{4}-\beta_{7}\left[\cos \left(\delta_{0}\right)-\cos \left(\delta_{0}+x_{1}\right)\right]+\frac{1}{T_{\mathrm{d} 0}} u_{2} \\
& y=C X=x_{1}+x_{3}
\end{aligned}
$$

where $\beta_{1}=1 / T_{H} ; \quad \beta_{2}=C / T_{H} ; \quad \beta_{3}=\omega_{0} / H ; \quad \beta_{4}=D / H \quad ;$ $\beta_{5}=\frac{\omega_{0}}{H} \frac{V_{\mathrm{S}}}{x_{d \sum}^{\prime}} \quad ; \quad \beta_{6}=\frac{x_{d \sum}}{T_{\mathrm{d} 0} x_{d \sum}^{\prime}} \quad ; \quad \beta_{7}=\frac{x_{\mathrm{d}}-x_{\mathrm{d}}^{\prime}}{T_{\mathrm{d} 0}} \frac{V_{\mathrm{S}}}{x_{d \Sigma}^{\prime}} \quad$; $X=\left[x_{i 1}, x_{i 2}, x_{i 2}, x_{i 4}\right]^{\mathrm{T}}=\left[\delta-\delta_{0}, P_{\mathrm{m}}-P_{\mathrm{m} \theta} \omega-\omega_{0}, E_{q}-E_{q 0}\right]^{\mathrm{T}} ; \quad \delta$ represent power angle; $\omega$ represent angular velocity; $P_{\mathrm{m}}$ represent mechanical 
power; $E_{q}^{\prime}$ represent the transient potential; $T_{H} \sum$ represent the control constant of the valve; $C$ represent power constant; $T_{\mathrm{d} 0}$ represent the time constant of the excitation winding; $x_{\mathrm{d}}$ represent $\mathrm{d}$-axis reactance; $x_{\mathrm{d}}^{\prime}$ represent $\mathrm{d}$-axis transient reactance; $x_{\mathrm{d} \Sigma}^{\prime}=x_{\mathrm{d}}^{\prime}+x_{\mathrm{T}}+x_{\mathrm{L}}, x_{\mathrm{T}}$ represent the reactance of the transformer, $x_{\mathrm{L}}$ represent the reactance of the transmission line; $H$ represent the moment of the inertia; $u_{1}=\mu-\mu_{0}, \mu$ represent the valve control, $\mu_{0}$ represent the initial valve control; $u_{2}=V_{f}-V_{f 0}, V_{f}$ represent the excitation control, $V_{f 0}$ represent the initial excitation control.

Under the transient and accident condition, such as the grounding of the transmission line, the values of $x_{\mathrm{d}}, x_{\mathrm{d}}^{\prime}, x_{\mathrm{d}}^{\prime} \Sigma$ will apparent change., The value of $D$ can't be measured at the same time. So in the equ. $1, \beta_{4}, \beta_{5}, \beta_{6}, \beta_{7}$ are the uncertain parameters.

Because there are too many uncertain parameters in the equ.1, it's difficult to calculate the values of $u_{1}$ and $u_{2}$ by the traditional robust methods, so the SEFERC will be adopting to realize the excitation and speed coordinate control.

Equ.21 can be translated into following equ.2, which is composed of "equivalent inner system" and "equivalent outer system".

$$
\begin{aligned}
\dot{x}_{1}= & x_{3} \\
\dot{x}_{2}= & -\beta_{1} x_{2}+\beta_{2} u_{1} \\
\dot{x}_{3 . o}= & -\beta_{5} \cos \left(\delta_{0}+x_{1}\right) x_{3}\left(x_{4}+x_{40}\right)-\beta_{1} \beta_{3} x_{2}-\beta_{4} x_{3 . o}+ \\
& \beta_{5} \beta_{6} x_{4} \sin \left(\delta_{0}+x_{1}\right)+\beta_{5} \beta_{7} \sin \left(\delta_{0}+x_{1}\right)\left[\cos \left(\delta_{0}\right)-\cos \left(\delta_{0}+x_{1}\right)\right]+ \\
& \beta_{1}\left(\beta_{3}-\beta_{30}\right) u_{1}-\frac{\left(\beta_{5}-\beta_{50}\right) \sin \left(\delta_{0}+x_{1}\right)}{T_{d 0}} u_{2}+\beta_{30} u_{1}-\frac{\beta_{50} \sin \left(\delta_{0}+x_{1}\right)}{T_{d 0}} u_{2} \\
= & f(\cdot)+\beta_{30} u_{1}-\frac{\beta_{50} \sin \left(\delta_{0}+x_{1}\right)}{T_{d 0}} u_{2} \\
y= & C X=x_{1}+x_{3}
\end{aligned}
$$

According to the DFL and linear $H_{\infty}$ control theory, the equ. 2 can be expressed by the following equ. 3 or equ. 4 . and the values of $u_{1}, v$ can be calculated.

$$
\begin{aligned}
& {\left[\begin{array}{l}
\dot{x}_{1} \\
\dot{x}_{2} \\
\dot{x}_{3} \\
\dot{x}_{3 . o}
\end{array}\right]=\left[\begin{array}{llll}
0 & 0 & 1 & 0 \\
0 & 1 & 0 & 0 \\
0 & 0 & 0 & 1 \\
0 & 0 & 0 & 0
\end{array}\right]\left[\begin{array}{l}
x_{1} \\
x_{2} \\
x_{3} \\
x_{3 . o}
\end{array}\right]+\left[\begin{array}{ll}
0 & 0 \\
\beta_{2} & 0 \\
0 & 0 \\
0 & 1
\end{array}\right]\left[\begin{array}{l}
u_{1} \\
v
\end{array}\right]} \\
& y=C X=x_{1}+x_{3}
\end{aligned}
$$

OR:

$$
\begin{aligned}
& \dot{X}=A X+B U \\
& y=C X=x_{1}+x_{3}
\end{aligned}
$$

In the optimal control theory, the value of $U$ is $U=R^{-1} B^{\mathrm{T}} P Z$. When the value of $v$ is confirmed, $u_{2}$ can be calculated by the following equ.5:

$$
u_{2}=\left(v-f(\cdot)-\beta_{30} u_{1}\right) T_{\mathrm{d} 0} / \beta_{50} \sin \left(\delta_{0}+x_{1}\right)
$$

Because there are uncertain parameters, such as $\beta_{4}, \beta_{5}$, $\beta_{6}, \beta_{7}$ in $f(\cdot)$, it is necessary to estimate the value of $f(\cdot)$. In order to estimate the value of $f(\cdot), f(\cdot)$ can be extended to the new variable $x_{30 . o} . x_{3 o . o}$ can be expressed by following equ.6.

$$
\begin{aligned}
& \dot{x}_{30 . o}=\beta_{5} \sin \left(\delta_{0}+x_{1}\right) x_{3}^{2}\left(x_{4}+x_{40}\right)+\beta_{1}^{2} \beta_{3} x_{2}+\beta_{5}\left\{\beta_{6} x_{4}+\right. \\
& \left.\left.\left.\left.\beta_{7}\left[\cos \oint_{0}\right)-\cos \oint_{0}+x_{1}\right)\right]\right\}\left[\beta_{6} \sin \left(\hat{\delta}_{0}+x_{1}\right)+2 x_{3} \cos \oint_{0}+x_{1}\right)\right]- \\
& \left.\beta_{5} \cos \oint_{0}+x_{1}\right)\left(x_{4}+x_{40}\right) x_{3 o}-\beta_{5} x_{30 . o}+\beta_{5} \sin ^{2}\left(\delta_{0}+x_{1}\right)- \\
& \left.\beta_{1} \beta_{2} \beta_{3} u_{1}+\frac{\beta_{5}}{T_{\mathrm{d} 0}}\left[\left(\beta_{7}-1\right) x_{3} \cos \oint_{0}+x_{1}\right)+\beta_{6} \sin \left(\delta_{0}+x_{1}\right)\right] u_{2} \\
& \left.=f_{1}(\cdot)-\beta_{1} \beta_{2} \beta_{3} u_{1}+\frac{\beta_{5}}{T_{d 0}}\left[\left(\beta_{7}-1\right) x_{3} \cos \oint_{0}+x_{1}\right)+\beta_{6} \sin \left(\delta_{0}+x_{1}\right)\right] u_{2} \\
& \quad=f_{1}(\cdot)-a u_{1}+b\left(x_{3}\right) u_{2}
\end{aligned}
$$

The equation, used to estimate the values of the $f(\cdot)$, can be expressed by following equ. 7 .

$$
\begin{aligned}
& {\left[\begin{array}{l}
\dot{x}_{1} \\
\dot{x}_{2} \\
\dot{x}_{3} \\
\dot{x}_{3 . o} \\
\dot{x}_{3 . o . o}
\end{array}\right]=\left[\begin{array}{lllll}
0 & 0 & 1 & 0 & 0 \\
0 & 1 & 0 & 0 & 0 \\
0 & 0 & 0 & 1 & 0 \\
0 & 0 & 0 & 0 & 1 \\
f_{1}(\cdot) &
\end{array}\right]\left[\begin{array}{l}
x_{1} \\
x_{2} \\
x_{3} \\
x_{3 . o} \\
x_{3 . o . o}
\end{array}\right]+\left[\begin{array}{ll}
0 & 0 \\
\beta_{2} & 0 \\
0 & 0 \\
0 & 0 \\
-a & b\left(x_{3}\right)
\end{array}\right]\left[\begin{array}{l}
u_{1} \\
u_{2}
\end{array}\right]} \\
& y=C X=x_{1}+x_{3}
\end{aligned}
$$

In the equ.7, $u_{1} 、 u_{2} 、 x_{1} 、 x_{2}$ is the present values, which should be measured by detecting hardware circuit. When the value $x_{3 \text { o.o }}$ (namely $\mathrm{f}($.$) is estimated, according the equ.7, the$ value of $u 2$ can be calculated. 


\section{SimULATION AND ANALYSIS}

In order to validate the effect of the SEFERC, the simulation of the excitation and speed coordinated control is performed. The parameters of the excitation and speed control mathematical model are as follows:

$\mathrm{Xd}=1.2, \mathrm{H}=11.6 \mathrm{~s}, \mathrm{Td}=1.15 \mathrm{~s}, \mathrm{TH}=0.35, \quad C=1, \quad x_{\mathrm{L}}=1.46$, $\mathrm{Pm} 0=0.32, D=3$ p.u.

Simulation 1: when the three phases short-circuit grounding appears at 1.1 second and disappears at 1.2 second, the comparison between the estimation value by STF and the real values of the state parameters and the extend state parameter $x_{3 . \text { o.o }}$ are shown as Figure 1.
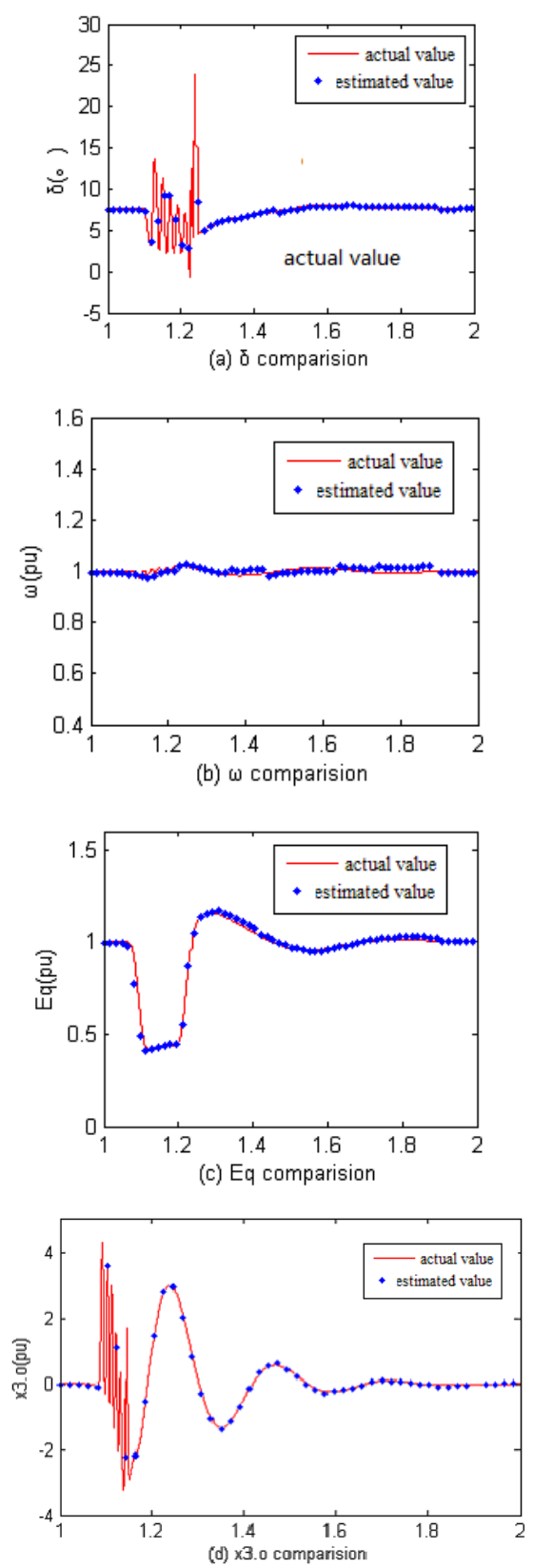

FIGURE I. THE COMPARISON BETWEEN THE REAL VALUE AND THE ESTIMATED VALUE OF THE STATE PARAMETER
As the shown of the figl.a to Figure 1.d, on the dynamic condition, the values of the state parameters in the ex-system can be estimated accurately by STF, which is the basis of the SEFERC.

Simulation 2: when the load is increased by $60 \%$ at 0.5 second and the SEFERC and the PID-PSS is adopting respectively, the change curves of the state and the control variables can be shown as Figure 2.a to Figure 2.d.

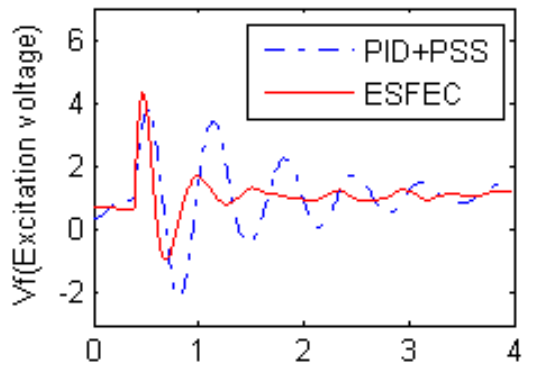

(a) $\vee f$

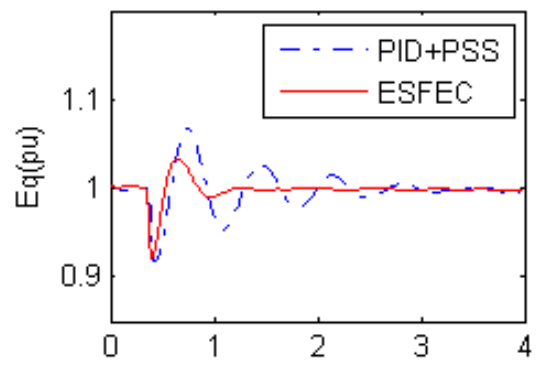

(b) Eq

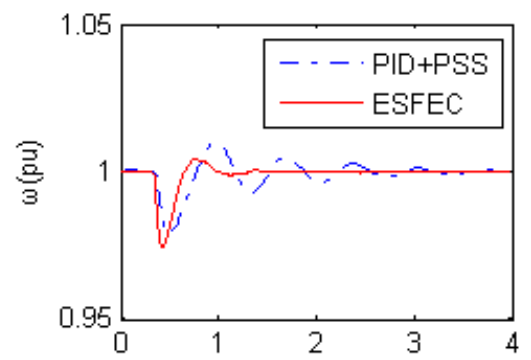

(c) $\omega$

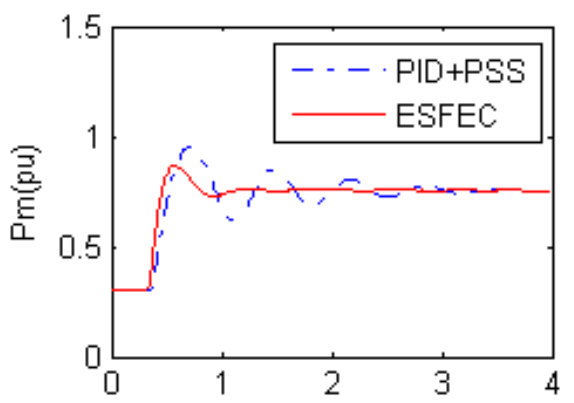

(d) $\mathrm{Pm}$

FIGURE II. CHANGE CURVE OF THE STATE AND THE CONTROL VARIABLES, WHEN THE SEFERC AND THE PID-PSS IS ADOPTING RESPECTIVELY 
As Figure 2.a to Figure 2.d shown, because the nonlinearity and uncertainty of the excitation and speed coordinated control system isn't considered in the PID-PSS control, the convergent speed of the state variables and the control speed are slow. On the contrary, because the values of the uncertain and disturbance part of the control system are estimated in the SEFERC, the excitation and speed coordinated control is accurate and the convergent speed is rapid. The SEFERC provides a new method for the nonlinear robust control.

\section{CONCLUSION}

In this paper, the state extending and filtering robust control (SEFERC) is proposed for the nonlinear MIMO system with many uncertain parameters, Compared with traditional robust control methods, which is based on the lyapunov stability theory, the SEFERC is based on the statistical principle and combine the DFL、 $H_{\infty}$ control method. In the SEFERC, the nonlinear uncertain parts of the state equation are extended to the new variables and estimated by nonlinear filtering method. When the values of the nonlinear parts of the system are known, combing the DFL, the nonlinear system can be changed into linear system. According to the $H_{\infty}$ control theory, the robust control of the linear system can be realized. Taking the excitation and speed coordinated control for example, results of the SEFERC simulation show that the values of the nonlinear uncertain parts of the system can be estimated accurately and the SEFERC can realize the robust control of the nonlinear uncertain system. Comparing with the PID-PSS control, the SEFERC can accelerate the convergence speed of the state variables and improve the control speed.

\section{ACKNOWLEDGMENT}

This research is financially supported by National Natural Science Foundation of China (Grant No. 51407196).

\section{REFERENCES}

[1] Wu Junyong. Coordinated Optimal Variable-Aim Nonlinear Control Strategy of Generator Excitation and Governor[J]. TRANSACT10NS OF CHINA ELECTROTECHNICAL SOCIETY, 2006,26(6):106-110.

[2] Huang Manlei, Song Ke-ming, Wei Zhida. Nonlinear H-two/H-infinity speed regulator for a diesel-generator set[J]. Control Theory \& Applications, 2009,26(8):873-878.

[3] Li Xiaocong, Cheng Shijie, Wei Hua, Sun Haishun. Multi Index Nonlinear Coordinated Control for TCSC and Hydro Turbine Generator Excitation and Governor [J]. Proceeding of the CSEE, 2004,24(11):2127.

[4] Liu Hui, Li Xiaocong, Wei Hua. Nonlinear Excitation Control for Generator Unit Based on NCOHF $[\mathrm{J}]$. Proceeding of the CSEE, 2007,27(1): 14-18.

[5] Wang Bing, Ji Haibo, Chen Huan, Xi Hongsheng. The Coordinated Passivity Techniques for the Excitation and Steam Valving Control of Generator [J]. Proceeding of the CSEE, 2004,24 (5) :14-18.

[6] Ma Jin, Xi Zairong, Mei Shengei, Lu Qian. Nonlinear Stabilizing Controller Design for the Steam Valving and Excitation System based on Hamiltonian Energy Theory[J]. Proceeding of the CSEE, 2002,22(5):8893.

[7] Wang Bing, Ji Haibo, Chen Huan, Xi Hongsheng. Nonlinear L2 Design for the Water Gate and Excitation Control of Hydro Turbine Generator based on Hamiltonian Energy Theory [J]. Electric Machines and Control,2006, 10(10):9-13.
[8] Guo Yajun, Wang Xiaofeng, Ma Dawei, Le Guigao. Application of Adaptive Backstepping Sliding Mode Controlling in Alternative Current Servo System of Rocket Gun[J]. ACTA ARMAMENTARil, 2011, 32(4): 493-497.

[9] Li Tianyun, Zhang Zhihua, Chen Feng, Zhang Yuhui. Design of Nonlinear Robust Controller of HVDC Power Transmission System Based on Extended State Observer and Terminal Sliding Mode Control [J]. Power System Technology,2012,36(10):190-195.

[10] Lan Zhou, Gan Deqiang, Ni Yixin, Xu Zheng. Decentralized Nonlinear Robust Adaptive Excitation Control Design for Power Systems[J]. Proceeding of the CSEE,2006,26(17):1-5.

[11] Zhao Lin. Nonlinear System Filtering Theory[M]. Beijing: National Dfense Industry Press,2012.

[12] Gu Zhifeng, Zhu Changqing, Shao Tianzhang, Wang Chuanchuan, An Qiaojing. Study on the AC tracking excitation based on the sensorless air-gap field oriented technology[J]. Electric Power Automatic Equipment,2011,10(31):52-56.

[13] Liu Ming, Zhou Donghua. Normalized residuals based strong tracking filter and its application[J]. Proceeding of the CSEE, 2005,25(2):71-75 